\title{
Prevalence and causes of musculoskeletal pain among middle school students in Sanandaj, Iran
}

Farshad Arghavani ${ }^{1}$, Homeira Alizadeh ${ }^{1}$, Khaled Rahmani ${ }^{2}$, Lale Rezai $^{1}$, Farzam Bidarpoor ${ }^{3}$, Foozieh Bigi ${ }^{3}$

\author{
Journal of Research \& Health \\ Social Development \& Health Promotion \\ Research Center \\ Vol. 6, No. 5, Nov \& Dec 2016 \\ Pages: $465-470$ \\ DOI: 10.18869/acadpub.jrh.6.5.465 \\ Original Article
}

1. Environmental Health Research Center, Kurdistan University of Medical Sciences, Sanandaj, Iran

2. Department of Epidemiology, School of Health, Shahid Beheshti University of Medical Sciences, Tehran, Iran

3. Social Determinants of Health Research Center, Kurdistan University of Medical Sciences, Sanandaj, Iran

Correspondence to: Homeira Alizadeh, Environmental Health Research Center, Kurdistan University of Medical Sciences, Sanandaj, Iran

Email: alizadeh homeira@gmail.com

Received: 25 Sep 2013

Accepted: 19 Nov 2013

How to cite this article: Arghavani F, Alizadeh H, Rahmani Kh, Rezai L, Bidarpoor F, Bigi F. Prevalence and causes of musculoskeletal pain among middle school students in Sanandaj. $J$ Research \& Health2016; 6(5): 465- 470.

\begin{abstract}
The prevalence of musculoskeletal pain among students is noticeable. Different studies have reported the prevalence rate above $40 \%$. The aim of this study was to investigate the prevalence of musculoskeletal pain among middle school students in Sanandaj, Iran, and discovering the causes of the pain among them. The study was performed on boy and girl students in secondary schools. 735 students in age range of 12 to 15 years was selected and some information about their school bags, chair type, and the number of hours watching TV or working on computer was obtained using a questionnaire. Weight, height, and BMI of students were measured. Body mapping revealed that musculoskeletal pain is appeared in the neck, shoulders, hands and wrists, back, elbows, back, hips, knees, and feet and ankles. The prevalence of musculoskeletal pain among schoolchildren was found to be $67 \%$. The prevalence of musculoskeletal pain had a significant relationship with sex, type of school bag, the number of hours watching TV and working on computer. The prevalence of musculoskeletal pain had no significant correlation with chair type used at school. The study showed that musculoskeletal pain is highly prevalent among the students. We need to teach the students to improve ergonomics and preventive behaviors in order to modify unsuitable conditions for students and teachers at school in taking steps. Risk factors for musculoskeletal pain in children are sex, type of school bags, and time spent watching TV and working on computer .
\end{abstract}

Keywords: Musculoskeletal Pain, School Bags, Student

\section{Introduction}

Musculoskeletal pain is the most common complaints of people in their life. No doubt musculoskeletal pain can develop serious musculoskeletal disorders (MSDs). MSDs injuries occur in the body's soft tissues such as muscles, tendons, joints, blood vessels, and nerves. MSDs gradually occur due to the accumulation of complications over months or years and in this point of view, are against the effects of sudden injuries such as bone fractures due to falling or neck injuries due to motor vehicle accident [1]. The prevalence of musculoskeletal pain is different at different ages. The spine is rapidly growing between age of 12-14 years and any stress to the spine reveals pain and discomfort 
[2]. Musculoskeletal pain is one of the most common causes visiting a doctor [3]. Students also experience spinal pain much sooner than it is imagined; hence they are vulnerable [4]. Musculoskeletal pain can cause numerous consequences such as lack of motivation, academic failure, and other learning problems in students [5].

According to several studies conducted in other countries, the prevalence of musculoskeletal pain has reported 40-60\%. Docrell et al. [6] showed overall prevalence of musculoskeletal pain among girl students as $80 \%$ and among boy students as 63 and expressed unsuitable carrying school bags by students as the major cause of pain. In another study Haza et al. [7] reported the prevalence of musculoskeletal pain as 40among 702 boy students at middle schools and carrying unfit school bags by the students was mentioned as the cause of pain. Buckle et al. [8] reported the prevalence of musculoskeletal pain as 49 among primary school students.

Students' exposure to unergonomic factors are the cause of musculoskeletal pain and can lead to several problems such as postural - structural problems and even learning disorders in students. Unfortunately, this issue has not been noticed in Iran and there are numerous unergonomic problems at schools. In Iran, only one study has been conducted on this subject. Shamsaldini et al. [9] investigated the prevalence of musculoskeletal pain among 340 primary and middle school students in Tehran. By using Nordic questionnaire, they reported the prevalence rate in shoulder 18.42 , neck 13.46 , and back 8.5 percent. In the aforementioned study, the causes of pain have not been studied. Considering that the living conditions of people in Tehran and their knowledge from various perspectives is different than the rest of the country, so the results cannot be generalized to other parts of the country.

Various causes contribute in students' musculoskeletal pain. Carrying unfit bags has the greatest role. Improper carrying school bag exposes the students to risk of postural problems and early fatigue as well. Increasing the weight of students' bag or backpack change the body posture and musculoskeletal system needs to respond properly in order to compensate for this stress [6]. In addition, carrying school bag on one shoulder or hand causes lateral curvature of the spine and increases the pressure on the opposite shoulder and hand and carrying school bag or backpack on both sides increase bending the trunk and forward the head posture and consequently musculoskeletal pain [10].

The static posture while working on computer or watching television, uncomfortable shoes, lack of proper seat, incompatibility of computer work stations with students' body size, and type of monitors are the main causes of musculoskeletal pain [11].

Any stress in physical and psychological form can change body posture in standing position and cause improper spinal alignment, and thus, the performance of disks between the vertebras that works like a buffer will disrupt [12]. According to the experts, inappropriate postural- structural adjustment will be a cause of musculoskeletal pain later in life. In addition, girls suffer postural structural problems more than boys because of physical inactivity [13-14].

In general, students musculoskeletal pain is due to frequent load carrying [15], inappropriate mobility [16], poor physical condition, and sedentary in recent decades $[17,18]$. It is obvious that more studies according to the epidemiological situation around Iran are felt to highlight the aspects of this issue. That is why this study aimed to assess the prevalence of musculoskeletal pain and determine some causes of pain among middle school students in Sanandaj.

\section{Method}

This cross sectional study was conducted among middle school students in Sanandaj. The sample size of 735 was calculated according to the results of previous studies using the prevalence rate of $49 \%$, confidence interval of $\% 99$, and precision of $5 \%$.

Stratified cluster sampling method was 
employed to select the subjects. So, Sanandaj city was divided into two areas (zone 1 and 2) as the strata and every school in each stratum was defined as a cluster. Then, random sampling was performed to choose the clusters and all students in the selected schools were included into the study.

We conducted the study on middle school students because of the critical period of physical growth of children in this age range. The second and third grade students were selected because of better understanding of the questionnaire compared with the first grade students.

The tool used in this study was a researcher-made questionnaire that its reliability and validity was tested by the researchers. 10 experts investigated the validity of the questionnaire and confirmed it after reformation. The test--test method was used for evaluating the reliability. In this case, 100 students completed the questionnaire twice with time interval of 2 weeks. Data analysis in SPSS-19 showed the correlation coefficient of 0.93 , which indicates a high reliability for the questionnaire.

The questionnaire included the questions in 4 parts. Demographic data including age, gender, school name, weight, height, seat type, and a brief history of background diseases which causes pain was recorded in the first part of questionnaire. Individual activities both inside and outside the school were asked in the second and the third part of questionnaire. In the fourth part, bags and carrying the bags (on one or both shoulders) were investigated. Students were asked to mark experienced musculoskeletal pain during the past 6 weeks especially in the neck, shoulder, and lower back by using body map that was designed for this purpose. At the same time, the student's height and weight were measured and recorded.

SPSS-19 was used for conducting descriptive and analytical statistical methods to analyze data.

Chi-square was used to test the independence of two qualitative variables. For comparing the quantitative data, one- way and two - way ANOVA were used. Nonparametric tests of Kruskal Wallis and Mann Whitney-u were applied for abnormal distributed data. The significance level was assumed at 0.05.

\section{Results}

Students' demographic characteristics are shown in Table 1.

Table1 Students'demographic characteristics

\begin{tabular}{llcccc}
\hline & & & & Weight $(\mathrm{kg})$ & Height $(\mathrm{m})$ \\
& & Frequency & $\%$ & Mean \pm SD & Mean \pm SD \\
\hline \multirow{3}{*}{ Age (year) } & 12 & 162 & 22 & $45.99 \pm 6.9$ & $1.44 \pm 5.2$ \\
& 13 & 250 & 34 & $48.46 \pm 7.1$ & $1.46 \pm 4.9$ \\
& 14 & 250 & 34 & $52.12 \pm 7.2$ & $1.51 \pm 4.7$ \\
\multirow{2}{*}{ Gender } & Girls & 390 & 53 & $50.36 \pm 8.4$ & $1.50 \pm 6.5$ \\
& Boys & 345 & 47 & $50.16 \pm 7.8$ & $1.48 \pm 4.9$ \\
\hline \multirow{2}{*}{ Educational zone } & Zone1 & 331 & 45 & $52.12 \pm 9.8$ & $1.51 \pm 6.3$ \\
& Zone2 & 404 & 55 & $50.99 \pm 8.9$ & $1.49 \pm 5.1$ \\
\hline \multirow{2}{*}{ Education grade } & Second Grade & 372 & 50.6 & $50.13 \pm 6.5$ & $1.50 \pm 5.7$ \\
& Third Grade & 363 & 49.4 & $54.27 \pm 6.7$ & $1.53 \pm 6.01$ \\
\hline
\end{tabular}

According to the obtained results, among 735 students surveyed, the magnitude of 492 students $(67 \%)$ reported musculoskeletal pain in at least one body part and 243 students (33\%) did not report any musculoskeletal pain. The overall prevalence rate indicates the percentage of students who reported pain in at least one body part. Table 2 shows the prevalence of musculoskeletal pain in 9 different parts of students' body.

According to Table 2, shoulder, lower back, neck, upper back, knees, foot and ankle, hand and wrist, hips, and elbows had the highest prevalence of musculoskeletal pain in sequence.

The results showed a statistically significant 
Table 2 The prevalence of musculoskeletal pain in different parts of students' body

\begin{tabular}{lcc}
\hline Pain & Frequency & $\%$ \\
\hline Shoulders & 370 & 50.4 \\
Lower back & 292 & 39.7 \\
Neck & 269 & 36.6 \\
Upper back & 198 & 27 \\
Knees & 150 & 20.4 \\
Foot and ankle & 149 & 20.3 \\
Hand and wrist & 95 & 13 \\
Tight & 69 & 9.4 \\
Elbow & 30 & 4.7 \\
\hline
\end{tabular}

Table 3 Factors associated with students' musculoskeletal pain

\begin{tabular}{|c|c|c|c|c|c|c|}
\hline & & \multirow{2}{*}{ Frequency } & \multirow{2}{*}{$\%$} & \multicolumn{2}{|c|}{ Musculoskeletal pain (\%) } & \multirow{2}{*}{ p-value } \\
\hline & & & & yes & no & \\
\hline \multirow{2}{*}{ Gender } & Girl & 390 & 53 & 70.3 & 29.7 & \multirow{2}{*}{0.04} \\
\hline & Boy & 345 & 47 & 65 & 35 & \\
\hline \multirow{3}{*}{ Bag school } & Backpack & 463 & 63 & 45.6 & 54.4 & \multirow{3}{*}{0.001} \\
\hline & Shoulder bag & 154 & 21 & 68.2 & 31.8 & \\
\hline & handbag & 118 & 16 & 63.2 & 36.8 & \\
\hline \multirow{2}{*}{ Seat type } & Table and bench & 441 & 60 & 64.8 & 35.2 & \multirow{2}{*}{0.334} \\
\hline & Seat handle & 294 & 40 & 67.4 & 32.6 & \\
\hline \multirow{4}{*}{$\begin{array}{l}\text { Working on } \\
\text { computer or } \\
\text { watching TV(hr) }\end{array}$} & $<1$ & 147 & 20 & 60.2 & 39.8 & \multirow{4}{*}{0.01} \\
\hline & $1-2$ & 147 & 20 & 63.2 & 36.8 & \\
\hline & $2-3$ & 191 & 26 & 91.6 & 8.4 & \\
\hline & $3<$ & 250 & 34 & 90 & 10 & \\
\hline
\end{tabular}

relationship between musculoskeletal pain and gender $(\mathrm{p}=0.04)$. A significant relationship was observed between musculoskeletal pain and the type of school bag $(\mathrm{p}=0.001)$. The findings also revealed a positive relationship between musculoskeletal pain and the number of hours of working on computer or watching TV $(p=0.01)$. No significant relationship was observed between seat type and the prevalence of musculoskeletal pain $(\mathrm{p}=0.334)$.

\section{Discussion}

The results showed that the mean height and weight of students were $1.48 \mathrm{~m}$ and $49.96 \mathrm{~kg}$ respectively. The prevalence of musculoskeletal pain in at least one part of the body among the students was $67 \%$. The prevalence of pain in shoulder was found to be $50.4 \%$, lower back $39.7 \%$, neck $36.6 \%$, upper back $27 \%$, knee pain $20.4 \%$, foot and ankle $20.3 \%$, hand and wrist $13 \%$, and thigh pain $9.4 \%$.

The prevalence of musculoskeletal pain among middle school students in Sanandaj was 22\% higher than the prevalence among students in Tehran [9]. The possible reason for this difference may be due to that students in Sanandaj are exposed to ergonomic risk factors associated with school more than students in Tehran.

In this study, the prevalence of musculoskeletal pain was $19.9 \%$ less than the prevalence rate reported by Haza et al. [7] in Saudi Arabia and $10 \%$ higher than the rate obtained by Rojan et al. in India [16]. Low ergonomic knowledge of parents, students and even teachers may be some reasons for similarities and contradictions of musculoskeletal pain prevalence among Iranian students rather than students in other countries.

According to the results, shoulder pain had the maximum prevalence of musculoskeletal pain and after that, were the body parts of lower back, neck, upper back; knees, feetlankles, hands $\backslash w r i s t s$, and thighs while the minimum rate belonged to elbows. In Shamsoddini et al. study [9], the highest prevalence of musculoskeletal pains was fond in shoulders, which is consistent with the present study. In 
this study, shoulder and neck pain prevalence was obtained more than the Shamsoddini et al. research. In this regard, in the research of Lyer et al. [1], the prevalence rate in shoulder, neck, and lower back are close to the findings of current study. Weak economic situation in Sanandaj families and paying enough attention to improving the students' health by parents in Tehran may be the possible reasons for the higher prevalence rate of musculoskeletal pain among the students in this study compared with the Shamsoddini et al. findings.

The findings showed that the students mostly used backpacks for carrying (63\%). This finding is similar to the results of other studies like Mirzaei et al. [15], Shamsoddini et al. [9], Rojan et al. [16], Shier et al. [5], Dockrell et al. [6], and Whitfield et al. [18].

The results revealed a significant difference between musculoskeletal pains and sex. Girl students reported more pain which is in agreement with the findings of other researches $[1,3,5-9,14,18,19,20]$.

High prevalence of musculoskeletal pain among girl students could be due to their weak and sensitive muscle tissues and, consequently, their improper spinal alignment which results in a disruption in buffering performance of intervertebral discs. Therefore according to experts, improper posture will be a cause of musculoskeletal pain later in life. Moreover, the lack of appropriate physical activity makes girls more vulnerable than boys to postural problems.

The results of the current study showed a significant relationship between the type of school bag used by students and the prevalence rate of musculoskeletal pains, so that the prevalence of musculoskeletal pains among students who used backpacks was found lower than the others. This finding is consistent with the results of other studies $[6,10,11,14$ $16,18,19]$. It seems carrying backpacks on both shoulders helps to maintain the proper posture and reduce the prevalence of musculoskeletal pains among the students as a result. However, studies indicated that students often carry the school bag on one side of shoulder. It is clear that carrying the school bag on one side for a long time increases lateral curvature of the spine and misbalances postures and finally, increases the potential of musculoskeletal pains.

In this study, there was a significant relationship between the numbers of hours of working on computer or watching TV and the prevalence of musculoskeletal pains. Students who watching TV or working on computer more than 2 hours a day stated more musculoskeletal pains. This finding was in agreement with the results obtained by Sheir et al. [5], Raju et al. [20], Hazza et al. [7] Whittfield et al. [18]. Poor posture while watching TV or lack of ergonomic conditions when working on a computer can be the possible reasons for this finding. Under such circumstances, tendons, muscles, and other parts of the musculoskeletal system get too tired and results in musculoskeletal pains.

Determining the prevalence ofmusculoskeletal pain in 9 areas of the body and investigating the relationship between types of seat used by students at school and the prevalence of musculoskeletal pains have been the strengths of the present study compared to other studies conducted in Iran and around the world.

Based on the obtained results in this study and similar studies, authorities in education departments and health national systems are required to pay attention to the prevalence of musculoskeletal pain issue among students.

No Specific limitation was observed in the implementation of the current study except for some complications in coordinating school authorities and local education administrations which were alleviated by assistance of health deputy of Kurdistan university of medical sciences and heads of education department.

\section{Conclusion}

The study results revealed some valuable information about students and ergonomics situation at schools of Iran. Gender, type of school bag, number of hours of working on computer or watching TV were detected as effective factors in creating musculoskeletal 
pain in middle school students. The middle school students in Sanandaj reported high prevalence of musculoskeletal pains in comparison with students from other parts of the country. In this regard, it is suggested for education authorities and parents to try to improve the ergonomic situation of schools and students and consider the principles of ergonomics at schools and at home as a priority.

\section{Acknowledgements}

The present research was conducted with the support of the student research committee of Kurdistan university of medical sciences. Further, the authors would like to express their sincere thanks to the educational authorities and students participated in this research.

\section{Contribution}

Study design: FA, KR

Data collection and analysis: FA, HA, KR, LR, FB, FB

Manuscript preparation: FA, HA, KR, LR, FB, FB

\section{Conflict of Interest}

"The authors declare that they have no competing interests."

\section{Funding}

The author (s) received no financial support for the research, authorship and/or publication of this article.

\section{References}

1- Lyer S. An ergonomic study of chronic musculoskeletal pain in school children. Indian J Pediatr2001; 8(1): 937-41. 2- Weir E. Avoiding the back-to-school backache. CMAJ2002; 17;167(6): 669.

3- Legg S, Cruz CO. Effect of single and double strap backpack on lung function. Ergonomic2004; 47(3): 318-23. 4- Leboef $\mathrm{C}$, Kuok O. What age does low back pain become a common problem? Spine2007; 23(2): 228-34. 5- Shier I, Kruse W, Brahman T. The association of backpack use and back pain in adolescents. Spine2003; 28(9): 922-30.

6- Dockrell S, Kanae C, Knaeffa E. Schoolbag weight and the effects of schoolbag carriage on secondary school student triniy college dublin. Spine2004; 38(1): 1-6

7- Al-Hazzaa HM. School backpack how much load to Saudi school boys carry on their shoulders. Saudi Med
J2006; 27(10): 1567-71.

8- Buckle P. Ergonomics and musculoskeletal disorders. Occup Med (Lond)2005; 55(3): 164-7.

9- Shamsaldini A, Helisal M, Dalvand H, Khatibi A. The investigation of prevalence of musculoskeletal symptoms and discomfort caused by them in student of Tehran Journal of Annals of Military and Health Sciences Research2011; 8(3): 33-5.

10- Venkatesan R, Rosmah R, Pedeliar R. The prevalence of computer-related musculoskeletal pain among college student. American Medical Journal2012; 3(1): 33-66.

11- Catherine C, Robin B. The prevalence of neck and upper extremity musculoskeletal symptoms in computer mouse users. Int J Ind Ergon2000; 26(3): 347-56.

12- Grimmer K. The relationship between cervical resting posture and neck pain. Phesioterapy2005; 82(1): 12-20.

13- Grimer K. Mmeasuring the endurance capacity of the cervical flexor muscle group. Australian Journal of Physiotherapy2007; 40(4): 251-8.

14- Legg SJ, Mahanty A. Comparison of five modes of carrying a load close to the trunk. Ergonomics 1986; 28(1): 1653-60.

15- Mirzai R, Ansari H, Khammari A. Survey of relationship between ergonomic and environmental classrooms and pain sensation in students. Journal of Mazandaran University of Medical Sciences2010; 12(5): 13-7.

16- Rojan P, Koti A. Ergonomic assessment and musculoskeletal health of the underprivileged school children in Pune, India. Health Promotion Perspectives2013; 3(1): 36-43.

17- Palumbo N, George B, Johnson A. The effect of backpack load carrying on dynamic balance as measured by limits of stability. Work2001; 16(2): 123-9.

18- Whittfield J, Legg S, Hedderley D. School bag weight and musculoskeletal symptoms in new Zealand secondary school. Applied Ergonomics2005; 36(2):193-8.

19- Laura P. Work-related musculoskeletal disorders. Journal of Electromyography and Kinesiology2004; 24(9):13-23.

20- Raju R. The influence of psychosocial factors on the occurrence of musculoskeletal disorders regarding VDT users. European Journal of Scientific Research2010; 43(2): 216-24. 